\title{
Performance Optimization of VoIP Calls over Wireless Links Using H.323 Protocol
}

\author{
Sajal K. Das ${ }^{1}$, Enoch Lee, Kalyan Basu ${ }^{1}$, Naveen Kakani ${ }^{2}$, and Sanjoy K. Sen ${ }^{3}$ \\ ${ }^{1}$ Center for Research in Wireless Mobility and Networking (CReWMaN) \\ Department of Computer Science and Engineering \\ The University of Texas at Arlington \\ Arlington, TX 76019-0015 \\ \{das,basu\}@cse.uta.edu \\ ${ }^{2}$ Nokia Research Center, 6000 Connection Dr, Irving, TX 75039 \\ Naveen.Kakani@nokia.com \\ ${ }^{3}$ Nortel Networks, P.O. Box 833858, Richardson, TX 75083 \\ sanjoy@ nortelnetworks.com
}

\begin{abstract}
Intelligent mobile terminals (or users) of next generation wireless networks are expected to initiate voice over IP (VoIP) calls using session set-up protocols like H.323 or SIP (Session Initiation Protocol). To guarantee service quality of such applications, the call set-up protocol should be robust against network impairment generated in wireless networks.

In this paper, we analyze the performance of the $\mathrm{H} .323$ call setup procedure over the wireless link. We used two call modes of operation for this analysis namely regular and fast connect.

The proposed model assumes that a transparent radio link protocol (RLP), but without RLP retransmission, be used for VoIP packets while non-transparent (regular) RLP be used for $\mathrm{H.323}$ control packets. Our analytical models prove that the VoIP call set-up performance can degrade significantly even for moderately high air-link frame error rates (FERs). Furthermore, the call setup performance can be improved significantly using the robust radio link layer such as RLP with the modifications proposed in our paper. The analytical results were validated by our experimental measurements.
\end{abstract}

Index Terms - Wireless networks, H.323 protocol, Link-level QoS, Radio link protocol (RLP) Performance optimization.

\section{INTRODUCTION}

Multimedia services like audio, video, text communications at a reasonable cost are becoming reality as the Global Information Infrastructure (GII) is gradually becoming reality. The quality of these services has to be maintained at a level better than the current networks to make GII success. To reduce cost and make the network seamless, convergence of all these services on a single infrastructure network is essential. The switching and routing technologies of the core networks are evolving towards IP, ATM, Frame Relay and optical routing technologies. This migration of the core network towards the

This work was partially supported by Texas Advanced Research Program grant TARP-003594-013 and Nortel Networks. This work was done when Kalyan Basu, Enoch Lee and Naveen Kakani were at Nortel Networks, Richardson, Texas. packet based transmission, has created the opportunity to migrate the traditional circuit switched based voice traffic to this IP core network using VOIP technology.

Indeed, toll quality voice over IP (VoIP) networks has recently played one of the key roles leading towards the convergence of the voice, video, and data communications industry. Although it has been shown that it is feasible to carry voice and call signaling messages over the Internet, providing commercial grade services to meet clients' expectation is yet to take off. There are still many technical issues and challenges that need to be resolved before this new concept of all-IP communication becomes a reality. Generally speaking, such a unified end-to-end IP network can have the following four benefits:

- Cost Reduction: The convergence of both voice and data traffic can improve network efficiency and reduce operation costs.

- Simplification: An integrated infrastructure that supports all forms of communication allows more standardization and reduces the network complexity.

- Consolidation: Since personnel are among the most significant expense elements in a network, any opportunity to combine operations, to eliminate points of failure, and to consolidate systems would reduce expense.

- Advanced Applications: Even though telephony is the basic application for voice over all IP network, the long term benefits are expected to be derived from multimedia and multi-service applications. For example, E-commerce solutions can combine WWW access to information with a voice call button that allows immediate access to a call center agent from personal computers.

With the advent of wireless technology, the integration of multimedia service with the wireless Internet is also inevitable. Currently Wireless and Internet technologies performs quite well within its own domain. However, the integration of wireless links to Internet exhibits considerable challenges

Unpredictability in the air-link conditions, such as rapid fad- 
ing, shadowing, and intermittent disconnections, affects the radio link such that the frame error rate (FER) can be as high as $10^{-1}$. This causes a serious quality degradation to the end users. In this paper, we investigate the impact of wireless link errors on the performance of H.323 signaling to set-up VoIP calls. ${ }^{1}$ In particular, estimate the average call set-up delay for various link error conditions. (The proposed model and analysis, uses the simple Direct Routing Call Model of H.323 protocol.) The model has the flexibility to be extended easily to other types of call models such as Gate-keeper routed call signaling. By analytical models, it is shown that the VoIP call set-up performance can degrade significantly even for moderately high air-link FER's. Therefore, we recommend that the H.323 call signaling messages be treated differently from the information (media) streams. We have also shown that the call set-up performance can be improved significantly using a more robust radio link layer such as the RLP. The laboratory experiments validate the analysis we proposed. The rest of the paper is organized as follows. Section 2 gives an overview of H.323 protocol while Section 3 presents the mathematical model to estimate the performance of H.323 and RTCP (real-time transport control protocol) signaling over wireless. In Section 4, the call set-up delay for the Fast Connect procedure is compared to the regular call set-up procedure. Section 5 describes some experiments conducted with Microsoft NetMeeting to validate our model. Section 6 presents the idea of a classifier to separate media streams and control messages, and Section 7 concludes the paper.

\section{OVERVIEW OF H.323 Protocol}

This section presents a brief introduction to H.323 [2] and RTP/RTCP [3] protocols. The H.323 standard provides a foundation for audio, video and data communications across IPbased networks. point-to-point and point-to-multipoint conferences. The standard specifies the interfaces between LANs and other networks and addresses call control, multimedia management, and bandwidth management methods .

The H.323 protocol uses the concept of channels to structure the information exchange between communication entities. A channel is a transport layer connection, which can be either $\mathbf{u}-$ nidirectional or bidirectional. Call setup delay is very large in H.323 for a regular call which involves multiple messages from its underlying protocols like H.225 [4] and H.245 [5]. This delay is rather prominent on a low bandwidth, high loss environment. It is further aggravated by the higher delay margin on the wireless access links. The fast call set-up method is an option specified in H.323 that reduces the delay involved in establishing a call and initial media streams.

The Real-Time Transport Protocol (RTP) is used in conjunction with H.323 to provide end-to-end data delivery services with real-time characteristics. RTP can handle interactive audio and video services over a connection less network. At present RTP/RTCP , along with UDP provides the bare-bone real-time services capability to IP network with minimum reliability.

The H.323 protocol defines four major components for a network-based communications system: Terminals, Gateways,

\footnotetext{
${ }^{1}$ The discussion on Session Initiation Protocol [1] is beyond the scope of this paper. For inter-networking between SIP and H.323, refer to [12].
}

Gate-keepers and Multipoint Control Units. The Terminals are the client end-points that provide real-time, two-way communications. All H.323 terminals must also support the H.245 protocol, which is used to negotiate channel usage and capabilities. Three other components are required in the architecture: (i) H.225 for call signaling and call set-up, (ii) Registration/Admission/Status (RAS): a protocol used to communicate with a Gate-keeper, and (iii) RTP/RTCP for sequencing audio and video packets.

A typical H.323 call set-up is a three-step process [2] which involves call signaling, establishing communication channel for signaling and establishing media channels.

In the first phase of call signaling, the H.323 client request$s$ permission from the (optional) Gate-keeper to communicate with the network. Once the call is admitted, the rest of the call signaling will proceed according to one of the several call models described in [2]. In this paper, we have chosen the Direct Routing Call Model. Figure 1 and Figure 2 describe the message flows in H.225 and H.245 respectively.

In the Direct Routing Call Model scenario, the two endpoints communicate directly instead of being registered with a Gatekeeper. As shown in Fig.1, Endpoint 1 (the calling endpoint) sends the H.225 Setup (1) message to the well-known Call Signaling Channel Transport Identifier of Endpoint 2 (the called endpoint) who responds with the H.225 Connect (4) message which contains an H.245 Control Channel Transport Address for use in H.245 signaling. The H.225 Call Proceeding (2) message is optional.

Once the H.245 Control Channel (unidirectional) has been established, the procedures of Recommendation H.245 are used for capability exchange and to open media channels, as shown in Fig. 2. The first H.245 message to be sent in either direction is TerminalCapabilitySet $(5,7)$ which is acknowledged by the TerminalCapabilitySetAck $(6,8)$ message. There can be an optional MasterSlaveDetermination procedure invoked at this stage to resolve the conflict, for example, between the two endpoints trying to open a bidirectional channel. The procedures of H.245 are then used to open logical channels for the various information streams $(9,11)$. The OpenLogicalChannelAck $(10,12)$ message returns the transport address that the receiving end has assigned to the logical channel. Both the H.225 and H.245 messages are transmitted over a reliable transport layer. Two different TCP sessions are established for H.225 and H.245 procedures.

After the media channel has been set-up, RTCP CNAME (canonical name) [3] are exchanged so that multiple media streams (e.g., audio and video) to the same user can be synchronized. This works reasonably well for wireline networks which have a frame error rate (FER) less than $10^{-8}$. However, significant performance degradation to end users may occur in wireless networks in which the FER can be as high as $10^{-1}$.

\section{Performance Analysis of H.323 Signaling over WIRELESS}

We will assume the simple call set-up message flows of H.323 as depicted in Figure 1 and Figure 2. The total call set-up delay will be the cumulative delay due to: 


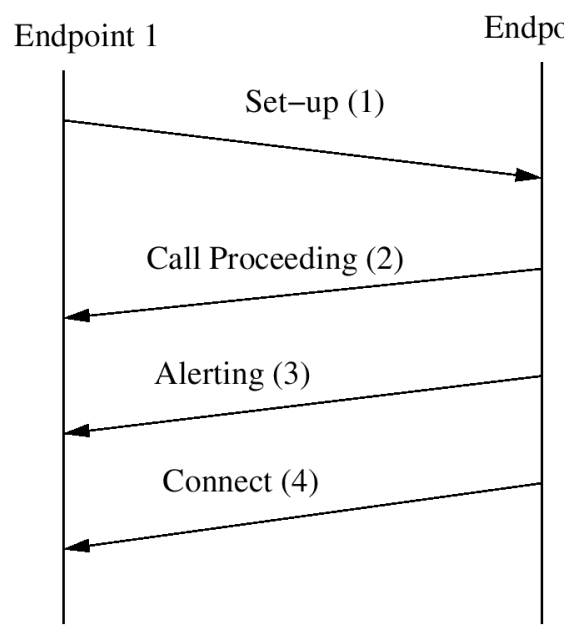

Fig. 1. H.225 Call signaling message flow

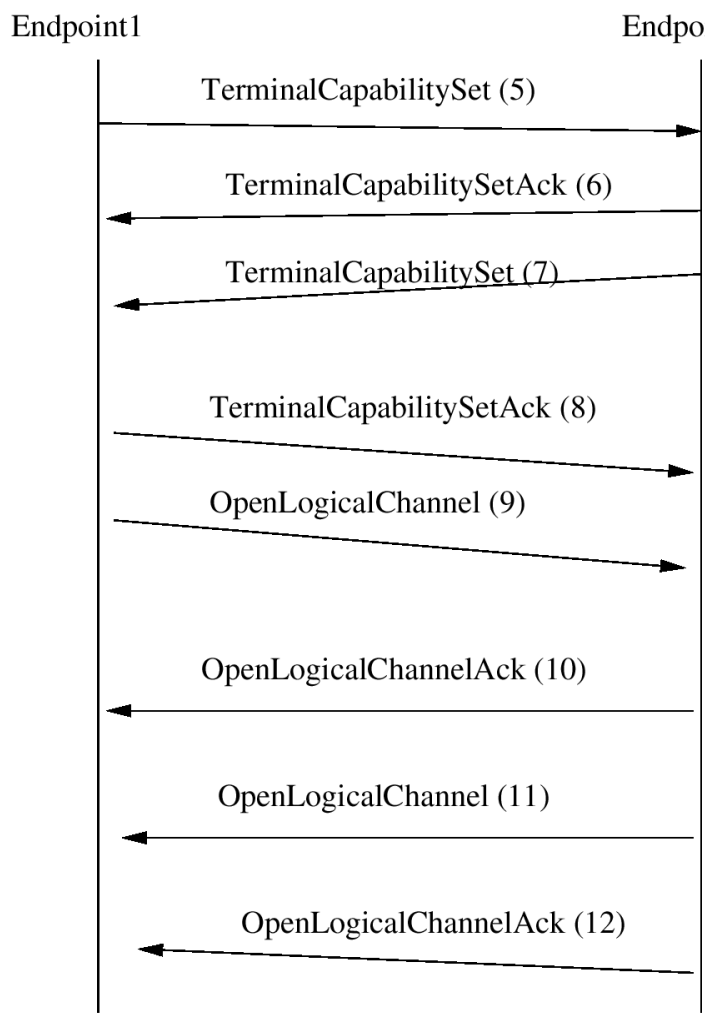

Fig. 2. H.245 message flow

(i) set-up time for two TCP sessions that include exchange of SYN, SYN-ACK, ACK messages;

(ii) successfully transmitting all H.225 and H.245 messages;

(iii) successfully receiving an RTCP CNAME message.

First, we present the analysis of RTCP packets based on mathematical models similar to those suggested in [6], [7] for CDMA networks. Next, we analyze H.323 call set-up flows and their interactions with TCP.

Some information in an RTCP packet are more important than others, e.g. CNAME and BYE. They are critical and need- ed to be reliably transmitted to guarantee user perceived quality of service (QoS) and the network performance. On the other hand, end-to-end retransmission is not recommended for realtime applications, like voice packets, to avoid delay variance. Local and controlled retransmission mechanisms at the wireless link is feasible for RTCP packets. Semi-reliable link layer retransmission mechanism like Radio Link Protocol (RLP) is used to reduce the air link FER and increases reliability. We introduce the retransmission mechanism of the proposed RLP scheme for cdma2000 for RTCP packets only to increase reliability of control packets.

When RLP at the receiving end finds a frame in error or a frame is missing, it sends back a NAK requesting for retransmission of this frame and starts a retransmission timer. When the timer expires for the first attempt, RLP resets the timer and sends back NAK again. This NAK triggers a retransmission of the requested frame from the sender. In this way, the number of attempts per retransmission increases with every retransmission trial. As noted in [6] the number of trials $(n)$ is usually less than 4 . We assume 3 trials in our analysis. We restrict the maximum retransmission time for a packet to be much less than the RTCP transmission interval $(T)$. An overdue RTCP packet is useless, since a new one will be generated and transmitted at certain period of time.

\section{A. Analysis of RTCP CNAME Packet Delay}

Let $p$ be the probability of a frame being in error in the air link. Let $K$ be the number of frames per RTCP packet transmitted over air and let $T \geq 5 \mathrm{sec}$ be the RTCP transmission interval. Let $D$ be the end-to-end frame propagation delay over the radio channel and its typical values is of the order of 100 msec. Let $\tau$ be the inter-frame time of of RLP and its typical value is of the order of $20 \mathrm{msec}$.

The packet loss rate without RLP is $q=1-(1-p)^{K}$. The packet loss rate with RLP is $q=1-\left(1-p(p(2-p))^{6}\right)^{K}$. This parameter will be used in the appropriate context of our analysis without notational ambiguity.

1) Without any Link Layer Reliability Mechanism: Assume a user just joined the RTP session. The average delay of receiving this CNAME packet indicates the waiting period for this user to be able to play the associated RTP streams properly. This is given as,

$$
\begin{aligned}
T_{1}= & D+(K-1) \tau+\left(\frac{T}{2}\right)(1-q)+\left(T+\frac{T}{2}\right) q(1-q) \\
& +\left(2 T+\frac{T}{2}\right) q^{2}(1-q)+\ldots \\
= & {\left[T \frac{1+q}{2(1-q)}\right]+D+(K-1) \tau } \\
= & T\left[\frac{2-(1-p)^{K}}{2(1-p)^{K}}\right]+D+(K-1) \tau
\end{aligned}
$$

2) With a Semi-reliable Link Layer Mechanism (RLP): For this analysis, let us define the following terms.

$X_{i j}=i$ th retransmission frame at the $j$ th retransmission trial received correctly at the destination.

$Y_{i j}=i$ th NAK frame at the $j$ th retransmission trial received correctly at the source. 
$C_{i j}=$ the first frame received correctly at the destination, being the $i$ th retransmission frame at the $j$ th retransmission trial.

$A_{j}=$ the missing frame not received correctly at the $j$ th retransmission trial.

$B_{j}=$ the missing frame not received correctly up to the end of the $j$ th retransmission trial.

Assume $P\left(X_{i} j\right)=P\left(Y_{i} j\right)=1-p$, where $p$ is the probability of a frame being in error in the air link. Therefore, if a frame is not received correctly at the $j$ th retransmission trial, then

$$
P\left(A_{j}\right)=\prod_{i=1}^{i=j}\left(1-P\left(X_{i j}\right) P\left(Y_{i j}\right)\right)=((2-p) p)^{j} .
$$

Since the probability that the frame is aborted after the $n$th retransmission trial is equivalent to the probability that the frame is not received correctly up to the end of the $n$th retransmission trial, this implies

$$
P\left(B_{n}\right)=p \prod_{j=1}^{n} P\left(A_{j}\right)=p(p(2-p))^{\frac{n(n+1)}{2}} .
$$

Thus,

$$
\begin{gathered}
P\left(C_{i j}\right)=P\left(B_{j-1}\right) P\left(X_{i j}\right) P\left(Y_{i j}\right) \prod_{k=1}^{k=j-1}\left(1-P\left(X_{k j}\right) P\left(Y_{k j}\right)\right) \\
=p(1-p)^{2}((2-p) p)^{\frac{j(j-1)}{2}+i-1} .
\end{gathered}
$$

Therefore the probability of transmitting a frame successfully over the RLC layer is given by

$$
P_{f}=1-P\left(B_{n}\right)=1-p(p(2-p))^{\frac{n(n+1)}{2}} .
$$

Then the probability of a packet loss is given by $q=1-P_{f}^{K}$, where $K$ is the number of the RLP frames in the RTCP packet.

Since retransmission is performed, the effective transport delay per frame increases from $D$ to $D^{\prime}$, where

$$
\begin{array}{r}
D^{\prime}=\frac{1}{P_{f}}\left[D(1-p)+\sum_{j=1}^{n} \sum_{i=1}^{j} P\left(C_{i j}\right)((2 j+1) D\right. \\
\left.\left.+\left[\frac{j(j+1)}{2}+i\right] \tau\right)\right]
\end{array}
$$

The ratio of the RTCP bandwidth usage when RLP is used to that without RLP is given as

$$
\frac{1}{P_{f}}\left[(1-p)+\sum_{j=1}^{n} \sum_{i=1}^{j} P\left(C_{i j}\right)(j(j-1)+2 i-1)\right]
$$

The delay experienced by an RTCP packet provided that RLP is used, is estimated as

$$
\begin{aligned}
D^{\prime \prime}= & \frac{1}{P_{f}^{k}}\left\{\sum_{l=0}^{k}\left(\begin{array}{c}
k \\
l
\end{array}\right)(1-p)^{k-l}\left(\sum_{j=1}^{n} \sum_{i=1}^{j} P\left(C_{i j}\right)\right)^{l}\right. \\
& \times\left[(D+(k-1) \tau)+\frac{l}{P_{f}} \sum_{j=1}^{n} \sum_{i=1}^{j} P\left(C_{i j}\right)\right. \\
& \left.\left.\times\left(2 j D+\left(\frac{j(j+1)}{2}+i\right) \tau\right)\right]\right\} \\
= & D+(k-1) \tau+\frac{k\left(P_{f}-(1-p)\right)}{P_{f}^{2}} \\
& \times\left(\sum_{j=1}^{n} \sum_{i=1}^{j} P\left(C_{i j}\right)\left(2 j D+\left(\frac{j(j+1)}{2}+i\right) \tau\right)\right)
\end{aligned}
$$

If RLP is used, the average delay for a newly joined member to receive the RTCP packet containing the CNAME item can be approximated by

$$
\begin{aligned}
T_{2}= & D^{\prime \prime}+\frac{T}{2}(1-q)+\left(T+\frac{T}{2}\right) q(1-q) \\
& +\left(2 T+\frac{T}{2}\right) q^{2}(1-q)+\ldots \\
= & T\left[\frac{1+q}{2(1-q)}\right]+D^{\prime \prime}=T\left[\frac{2-P_{f}^{K}}{2 P_{f}^{K}}\right]+D^{\prime \prime}
\end{aligned}
$$

\section{B. H.323 Call Set-up Message Delay Analysis}

Since H.225 and H.245 messages are carried over TCP, an analysis of TCP transport delay over wireless will lead to an insight to the of $\mathrm{H} .323$ call set-up delay performance. We have assumed a fixed radio channel bandwidth of $9.6 \mathrm{Kbps}$. The two endpoints are assumed to be in close proximity, hence any wireline network delay is assumed to be negligible. The following assumptions are made about the end-to-end TCP sessions carrying the $\mathrm{H} .323$ messages :

1) TCP is assumed to operate in an interactive mode (this is valid since most of the transactions consist of a single message and its acknowledgement).

2) The Delayed Acknowledgement mode of TCP operation is turned off. This implies that every TCP data packet will be acknowledged.

3) TCP always times outs whenever a packet is lost (i.e., it never does fast retransmit). This is valid due to the interactive nature of the transactions where an endpoint after transmitting an H.323 message (e.g., TerminalCapabilitySet) typically waits for its acknowledgement (e.g., TerminalCapabilitySetAck) before transmitting the next H.323 message.

4) The one way delay for the message path is assumed to be $D \approx 100 \mathrm{msec}$. Hence, the round trip delay is $200 \mathrm{msec}$.

5) The initial TCP round-trip timer (RTO) value is assumed to be exactly equal to the round-trip delay. Subsequent variation of RTO is as specified for TCP in [8].

6) The TCP packet sizes for each H.323 message is obtained from the actual H.225 and H.245 packets captured using a protocol analyzer. 
7) Assume for simplicity that if initial capability exchange or master-slave determination procedures fail, no retry should be issued as opposed to the standard that suggest$s$ at least two additional retransmissions before the endpoint abandon the connection attempt.

Following Karn's algorithm for TCP timer back-off, the $R T O$ is multiplied by a constant factor after each retransmission due to time-out. Hence, $R T O_{i+1}=c * R T O_{i}$ where $R T O_{i}$ is the $i$ th TCP retransmission timer. This causes $R T O$ to grow exponentially after each retransmission. We let $c=2$ as it is most commonly implemented. Initially $R T O=100 \mathrm{msec}$ as assumed previously. Hence $R T O_{1}=2 * 100 \mathrm{msec}, \ldots, R T O_{i}=$ $2^{i} * 100 \mathrm{msec}$. Furthermore, TCP will not allow infinite number of retransmissions. Hence, if TCP retransmission succeeds, say after $N_{m}$ attempts (without loss of generality we assume $N_{m}=$ 10), the average delay to transmit a TCP packet is:

$$
\begin{array}{r}
T^{\prime}+R T O_{1}+R T O_{2}+\ldots .+R T O_{m} \\
=T^{\prime}+\left(2^{N_{m}+1}-2\right) * 100
\end{array}
$$

where $T^{\prime}$ is the end-to-end propagation delay of the TCP packet. We shall establish the average delay for successful transmission of a TCP packet both with and without radio link reliable transmission scheme (RLP). We will then compare the average total H.323 call set-up delay with and without RLP.

For the purpose of our analysis, we stipulate in the following that the packet loss rate is $q<0.5$. Indeed, if packet loss rate is as high as $50 \%$, wireless application users may not be able to enjoy any proper services over the air-link. Recall that $D(100 \mathrm{msec})$ and $(20 \mathrm{msec})$ are the end-to-end frame propagation delay over the radio channel and the inter-frame time, respectively.

1) Average TCP Packet Transmission Delay Without RLP:

The TCP packet loss rate is $q=1-(1-p)^{k}$, where $p$ is the probability of a frame being in error in the air link and $k$ is the number of air-link frames contained in a TCP segment. The probability of successfully transmitting a TCP segment is (1 $q)+(1-q) q+\ldots+(1-q) q^{N_{m}-1}=1-q^{N_{m}}$. Using Equation (5), we obtain the average delay for successfully transmitting a TCP segment with no more than $N_{m}$ retransmission trials as,

$$
\begin{aligned}
T N= & \frac{1}{1-q^{N_{m}}}[(1-q)(D+(k-1) \tau) \\
& +(1-q) q(3 D+(k-1) \tau)+\ldots \\
& \left.+(1-q) q^{N_{m}-1}\left(\left(2^{N_{m}}-1\right) D+(k-1) \tau\right)\right] \\
= & (k-1) \tau+\frac{1-q}{1-q^{N_{m}}} D\left(1+\frac{4 q}{1-2 q}-\frac{q}{1-q}\right) \\
& +\frac{1-q}{1-q^{N_{m}}} D\left(\frac{q^{N_{m}}}{1-q}-\frac{2^{N_{m}+1} q^{N_{m}}}{1-2 q}\right) \\
= & (k-1) \tau+\frac{D}{\left(1-q^{N_{m}}\right)(1-2 q)} \\
& +\frac{1-q}{1-q^{N_{m}}} D\left[\frac{q^{N_{m}}}{1-q}-\frac{2^{N_{m}+1} q^{N_{m}}}{1-2 q}\right]
\end{aligned}
$$

The TCP packets are going to carry the H.323 control messages in the payload. Hence, the total call set-up delay is the cumulative addition of the delays for transmitting all the H.323 call set-up messages and the RTCP:CNAME packet.
The total delay without RLP is given as $T_{n o R L P}=$ $\sum_{i=1}^{N_{m}} T N_{i}+T C$, where, $T N_{i}$ is the average delay given by $\mathrm{E}$ quation (6) for the $i$ th TCP segment (carrying one of the H.323 control messages in the payload) and $T C$ is the average delay to receive the first RTCP:CNAME packet after joining the session.

2) Average TCP Packet Transmission Delay with RLP: We first evaluate the average delay to transmit a TCP segment (all $k$ frames) successfully given that the radio link layer guarantees the transmission of each frame. This is given as

$$
\begin{aligned}
D^{\prime}= & \frac{1}{P_{f}^{k}}\left\{\sum_{l=0}^{k}\left(\begin{array}{l}
k \\
l
\end{array}\right)(1-p)^{k-l}\left(\sum_{j=1}^{n} \sum_{i=1}^{j} P\left(C_{i j}\right)\right)^{l}\right. \\
& \times\left[(D+(k-1) \tau)+\frac{l}{P_{f}} \sum_{j=1}^{n} \sum_{i=1}^{j} P\left(C_{i j}\right)\right. \\
& \left.\left.\times\left(2 j D+\left(\frac{j(j+1)}{2}+i\right) \tau\right)\right]\right\} \\
= & D+(k-1) \tau+\frac{k\left(P_{f}-(1-p)\right)}{P_{f}^{2}} \\
& \times\left(\sum_{j=1}^{n} \sum_{i=1}^{j} P\left(C_{i j}\right)\left(2 j D+\left(\frac{j(j+1)}{2}+i\right) \tau\right)\right)
\end{aligned}
$$

where $n=3$ is the maximum number of RLP retransmission trials and

$$
P_{f}=1-p+\sum_{j=1}^{n} \sum_{i=1}^{j} P\left(C_{i j}\right)=1-p(p(2-p))^{\frac{n(n+1)}{2}}
$$

as shown in Section 3.1. Recall also that the packet loss rate is given by $q=1-P_{f}^{K}$. Thus the average delay to transmit a TCP segment successfully is given by:

$$
\begin{aligned}
T R= & \frac{1}{1-q^{N_{m}}}\left[(1-q) D^{\prime}+(1-q) q\left(2 D+D^{\prime}\right)+\ldots\right. \\
& \left.+(1-q) q^{N_{m}-1}\left(\left(2^{N_{m}}-2\right) D+D^{\prime}\right)\right] \\
= & D^{\prime}+\frac{2 D_{q}(1-q)}{1-q^{N_{m}}}\left[1+\frac{4 q\left(1-(2 q)^{N_{m}-2}\right)}{1-2 q}\right. \\
& \left.-\frac{q\left(1-q^{N_{m}-2}\right)}{1-q}\right]
\end{aligned}
$$

When the air-link FER is very high, it is possible that too much of RLP retransmissions will cause enough delay that TCP's retransmission timer always times out. In those cases, the situation degenerates to the case described in Section 3.1.1. Thus the average call set-up delay with RLP is given by

$$
T_{R L P}=\sum_{i=1}^{N_{m}} T_{i}+T C, \text { where } T_{i}=\min \left\{T R_{i}, T N_{i}\right\} .
$$

\section{Average H.323 CAll SeT-UP DElay}

Let us now compute the average call set-up delay for a regular H.323 procedure (as described in Section 2) and a Fast Connect procedure described in [2].

The models presented in the previous section implicitly implies that an average regular H.323 call set-up delay increases 
exponentially as FER increases. Three major factors contribute to the delay - the number of message exchanges, size of message exchanges, and the number of TCP sessions set-up. Reduction of any of these factors results in shorter call set-up delay for any H.323 call. The Recommendation H.323 provides ways for addressing this or similar issues. This include encapsulation of H.245 messages within H.225 messages called Tunneling, and the Fast Connect procedure. In order to conserve resources, either or both the mechanisms synchronize call signaling and control, and reduce call set-up time. These mechanisms can be invoked by the H.323 calling endpoint. In this section, we will investigate the call set-up delay incurred by the Fast Connect procedure only. See Appendix for a brief introduction to the Fast Connect procedure.

H.323 endpoints may establish media channels in a call using either the regular procedures defined in Recommendation H.245 or the so called Fast Connect procedure. The Fast Connect procedure allows the endpoints to establish a basic pointto-point call with as few as one round-trip message exchange, enabling immediate media stream delivery upon call connection.

\section{A. Fast Connect Procedure}

As the number and size of TCP messages (H.225 signaling messages) increase, the cumulative transmission time for those messages increases correspondingly. Therefore the size and the number of the TCP packets affect the call set-up time. As an example, Table 1 shows the size ${ }^{2}$ of the H.225 signaling for Fast Connect procedures. We assume a TCP segment is carried in one IP packet and frames imply air-link frames. TCP and IP headers' sizes are of 20 octets each. For the Setup + fastStart message, we assume 3 openLogicalChannel structures included; whereas in the Connet + fastStart case, 1 openLogicalChannel is chosen for media exchanges by called endpoint.

TABLE I

Mess age Sizes associated With the H.225 Fast Connect PROCEDURE

\begin{tabular}{||c|c|c|c||}
\hline \hline Messages & $\begin{array}{c}\text { Payload } \\
\text { size }\end{array}$ & $\begin{array}{c}\text { \# frames } \\
(9.6 \mathrm{Kbps})\end{array}$ & $\begin{array}{c}\text { \# frames } \\
(19.2 \mathrm{Kbps})\end{array}$ \\
\hline $\begin{array}{c}\text { Setup + } \\
\text { fastStart }\end{array}$ & 599 octet & 30 & 15 \\
\hline Alerting & 97 octet & 7 & 4 \\
\hline $\begin{array}{c}\text { Connect }+ \\
\text { fastStart }\end{array}$ & 280 octet & 15 & 8 \\
\hline RTCP packet & 120 octet & 8 & 4 \\
\hline \hline
\end{tabular}

\section{B. Regular Procedure}

Based on the H.323 call model, the overall delay for call setup is computed and shown below. As the number and size of TCP messages (signaling and control messages) increase, the cumulative transmission time for these messages increases correspondingly. Therefore, the size and the number of the TCP

\footnotetext{
${ }^{2}$ The analysis includes the TCP set-up messages, SYN, SYN-ACK, ACK, although they are not shown in Table 1.
}

packets affect the call set-up time and media synchronization. Data in Table 2 shows the size of the signaling and control messages as an example. Here TCSet denotes 'Terminal CapabilitySet' and OC denotes 'OpenlogicalChannel'.

TABLE II

MESSAGE SIZES ASSOCIATED WITH THE REgULAR CALL SET-UP PROCEDURE

\begin{tabular}{||l|l|l|l||}
\hline \hline Messages & Payload size & $\begin{array}{l}\text { \# frames } \\
9.6 \mathrm{Kbps}\end{array}$ & $\begin{array}{l}\text { \# frames } \\
19.2 \mathrm{Kbps}\end{array}$ \\
\hline Setup -H.225 & 254 octet & 14 & 7 \\
\hline Alerting $-\mathrm{H} .225$ & 97 octet & 7 & 4 \\
\hline Connect $-\mathrm{H} .225$ & 165 octet & 10 & 5 \\
\hline TCSet $-\mathrm{H} .245(\mathrm{x} 2)$ & 587 octet $(\mathrm{x} 2)$ & $29(\mathrm{x} 2)$ & $15(\mathrm{x} 2)$ \\
\hline TCSetAck $-\mathrm{H} .245(\mathrm{x} 2)$ & 71 octet $(\mathrm{x} 2)$ & $6(\mathrm{x} 2)$ & $3(\mathrm{x} 2)$ \\
\hline OC $-\mathrm{H} .245(\mathrm{x} 4)$ & 115 octet $(\mathrm{x} 4)$ & $8(\mathrm{x} 4)$ & $4(\mathrm{x} 4)$ \\
\hline OCAck $-\mathrm{H} .245(\mathrm{x} 4)$ & 64 octet $(\mathrm{x} 4)$ & $5(\mathrm{x} 4)$ & $3(\mathrm{x} 4)$ \\
\hline RTCP packet & 120 octet & 8 & 4 \\
\hline \hline
\end{tabular}

Figure 3 and Figure 4 compare the average call set-up delays associated with a regular connect procedure and a Fast Connect procedure for a 9.6 Kbps and 19.2 Kbps channels, respectively. Each procedure is further evaluated with and without support from RLP over the error-prone wireless channel. It can be observed that FastConnect with RLP support provides the minimum call set-up delay. Furthermore, the call set-up delay for Fast Connect procedure is consistently below 5 seconds and 4 seconds for $9.6 \mathrm{Kbps}$ and $19.2 \mathrm{Kbps}$ channels, respectively, if FER is less than 9\%. This is a few seconds faster than the corresponding regular call set-up procedure.

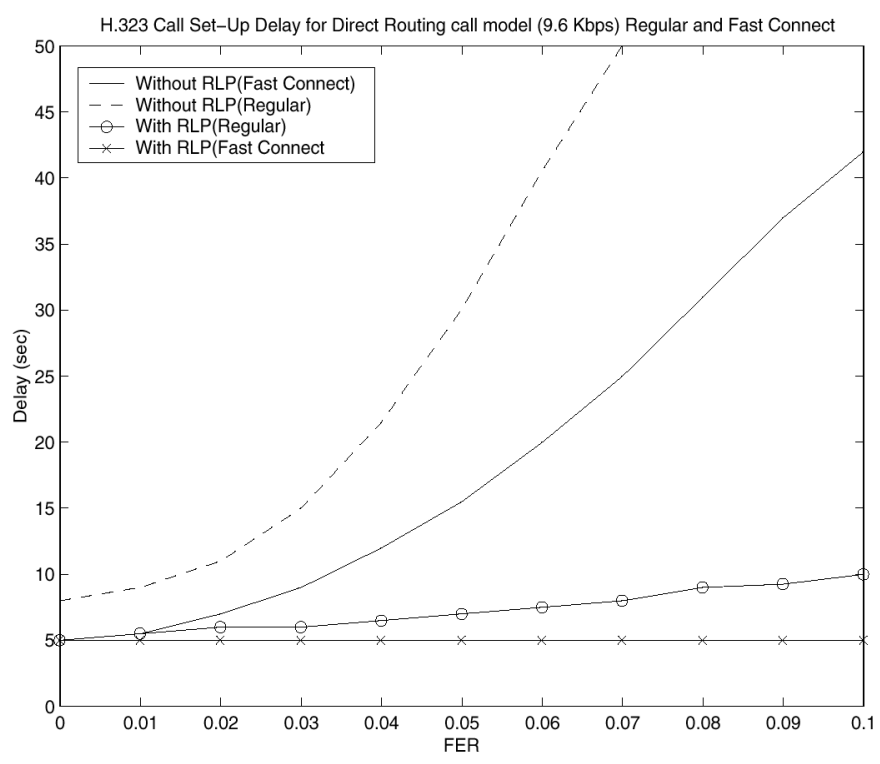

Fig. 3. Call set-up delay in a $9.6 \mathrm{Kbps}$ channel

\section{EXPERIMENTAL VERIFICATION}

Experiments for call setup delay at various FER rates between 1-10\% over the Wireless Link Emulator (WLE) were conducted using Microsoft NetMeeting. An end-to-end Microsoft NetMeeting VoIP session from Endpoint A (Caller) to 


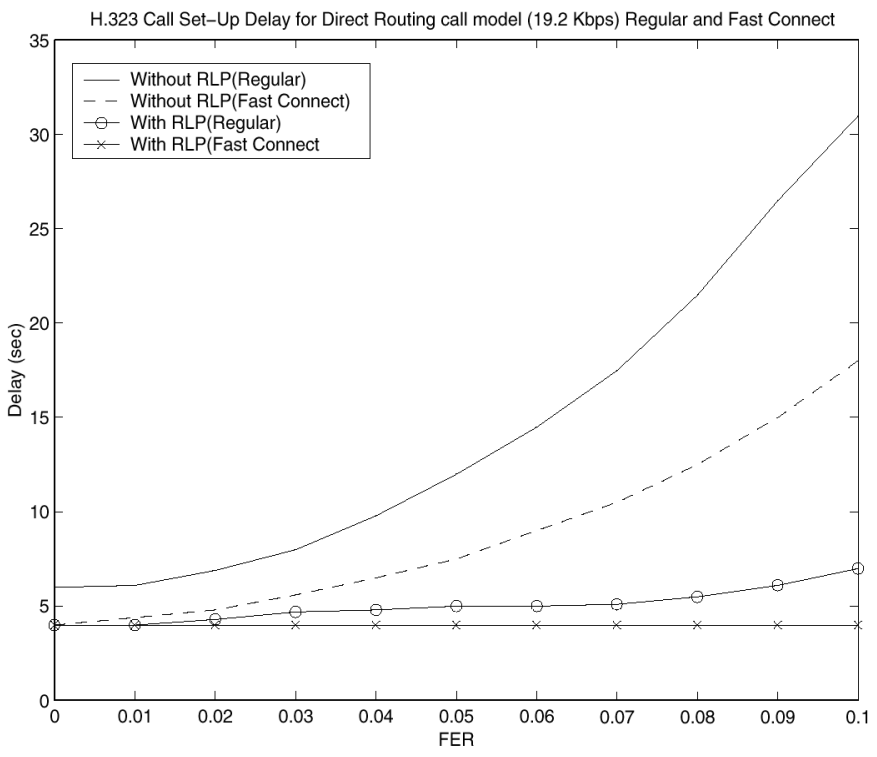

Fig. 4. Call set-up delay in a 19.2 Kbps channel

Endpoint B (Callee) over the WLE was created. NetMeeting 3.01 uses the H.323v2 call signaling (Q.931/H.225/H.245 over TCP) to set up VoIP session and the default Microsoft codec G.723.1, $8 \mathrm{kHz}$ Mono, $6400 \mathrm{Bit} / \mathrm{s}$ for audio compression. In these experiments, the call is considered successful only when the voice path is cut through (i.e., Endpoint B can hear the caller's voice). In addition, the maximum amount of time the called party waited for voice cut-through is 2 minutes, after which the call was marked as unsuccessful.

Two sets of experiments were conducted, one with the channel bandwidth fixed at $9.6 \mathrm{Kbps}$ and the others at $19.2 \mathrm{Kbps}$. Thirty delay samples were collected at each FER with RLP and without RLP. Then, the sample mean was computed (see Figure 5 and Figure 6). The success rate at each FER is shown in Table 3.

With RLP turned on, the result of the call setup success for both $9.6 \mathrm{Kbps}$ and $19.2 \mathrm{Kbps}$ is perfect, at $100 \%$ success. With RLP turned off, at $1 \%$ air-link FER for $9.6 \mathrm{Kbps}$ and $1-2 \%$ airlink FER for $19.2 \mathrm{Kbps}$, the call setup success rate is $100 \%$. However, as the air-link FER rate increases, the average call setup delay increases (Figures 5 and 6 ) and the success rate declined (Table 3 ).

TABLE III

CALL SET-UP SUCCESS RATE VS. FER

\begin{tabular}{||c|c|c|c|c||}
\hline \hline FER & \multicolumn{4}{|c||}{ Call Set-up Success Rate } \\
\hline & $\begin{array}{c}9.6 \mathrm{Kbps} \\
\text { (w/ RLP) }\end{array}$ & $\begin{array}{c}19.2 \mathrm{Kbps} \\
\text { (w/ RLP) }\end{array}$ & $\begin{array}{c}9.6 \mathrm{Kbps} \\
\text { (w/o RLP) }\end{array}$ & $\begin{array}{c}19.2 \mathrm{Kbps} \\
\text { (w/o RLP) }\end{array}$ \\
\hline $1 \%$ & $100 \%$ & $100 \%$ & $100 \%$ & $100 \%$ \\
\hline $2 \%$ & $100 \%$ & $100 \%$ & $93 \%$ & $100 \%$ \\
\hline $3 \%$ & $100 \%$ & $100 \%$ & $83 \%$ & $93 \%$ \\
\hline $4 \%$ & $100 \%$ & $100 \%$ & $47 \%$ & $93 \%$ \\
\hline $5 \%$ & $100 \%$ & $100 \%$ & $30 \%$ & $87 \%$ \\
\hline $8 \%$ & $100 \%$ & $100 \%$ & $0 \%$ & $40 \%$ \\
\hline $10 \%$ & $100 \%$ & $100 \%$ & $0 \%$ & $23 \%$ \\
\hline \hline
\end{tabular}

Fig. 6. Comparison of NetMeeting and Theoretical model Call Set-up delay vs. FER over 19.2 Kbps radio channel

A fast call set up time is considered a significant step towards providing QoS. Previous sections illustrate that it is advantageous to transmit $\mathrm{H} .323$ call set-up messages and RTCP packets over air link with RLP. Especially in the case of using VoIP client that requires exchanges of several signaling and control messages before connection. For instance, the call set up procedure for H.323 involves exchanges of $\mathrm{H} .225$ and $\mathrm{H} .245$ messages. As a consequence, the total delay to replay a media is the sum of the delays experienced by each of the messages. Hence the usage of RLP, with either the regular or Fast Connect call set-up procedure, enhances services without sacrificing a great deal of the limited bandwidth. 


\section{H.323 SUB-FlOW ClaSSIFICATION FOR VoIP}

In the previous two sections, we have demonstrated that the performance of the $\mathrm{H} .323$ protocol to set-up a multimedia call can be poor even under moderately high air-link FER's. This is primarily due to the poor performance of TCP under high FER without the support of a local retransmission mechanism over the wireless link, such as the RLP. Hence, there is a need to treat the H.323 media streams for interactive traffic (particularly, VoIP) differently from the H.323 control packet streams (e.g., H.225, H.245, RTCP messages). Since the VoIP packet$\mathrm{s}$ can sustain higher FER than the control traffic, it is recommended that transparent RLP (i.e. no RLP retransmission) be used for VoIP packets while non-transparent (regular) RLP be used for H.323 control packets. Also, due to the interactive nature of voice, VoIP packets should get higher priority over any in-band H.323 control packets within the session.

Two types of sub-flows requiring differentiation have been identified for VoIP service using H.323. These are:

- H.323 control packets including H.225, H.323 and RTCP These packets need higher reliability for better performance. They have less stringent delay requirements compared to the media packets.

- H.323 media packets carried by RTP These packets have lower reliability requirements than the control packets, but have stringent delay requirements.

We propose a classifier to classify the sub-flows in wireless VoIP implementation using H.323 protocol. The preliminary functional requirements of this classifier is in Figure 7. We recommend that this classifier be used in conjunction with RLP to improve the performance of VoIP over wireless.

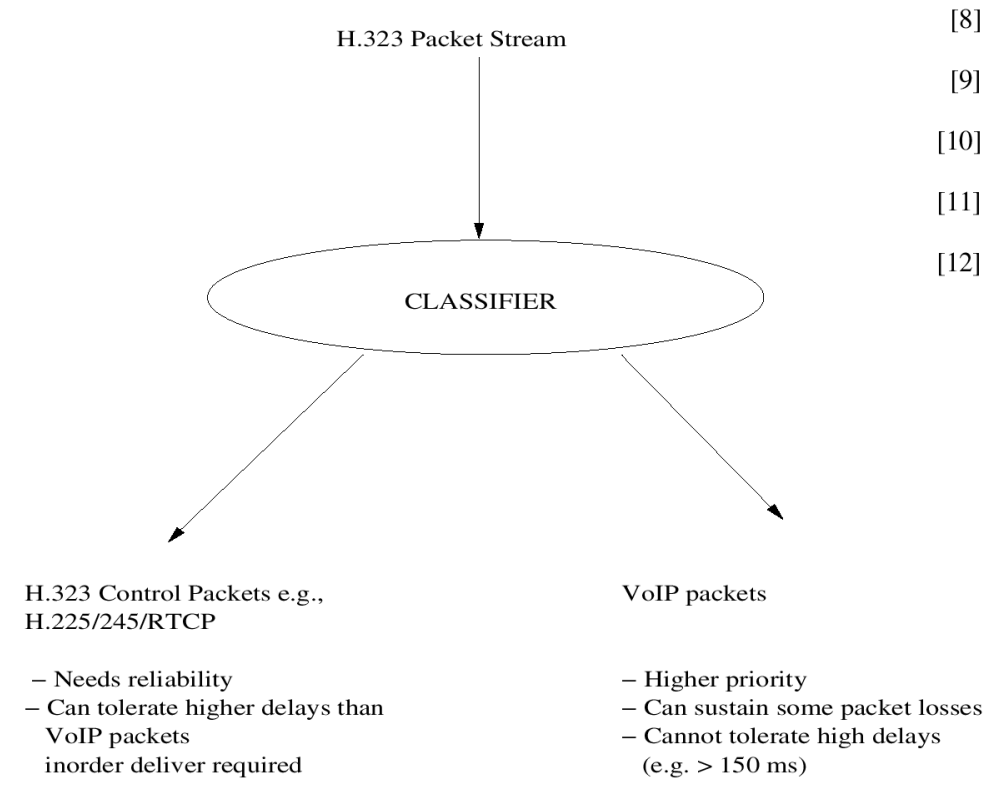

Fig. 7. H.323 Sub-flow Classes requiring different treatment

\section{CONCLUSION}

In this paper we proposed an analytical model to estimate the performance of the $\mathrm{H} .323$ call set-up procedure for wireless links under the regular and the Fast Connect modes of operations. We showed that the call set-up time with a lower layer retransmission mechanism (such as RLP) significantly improves the performance of VoIP over wireless. The analytical model is validated by taking Lab measurements using wireless link emulator and Microsoft's NetMeeting. Finally, we proposed the concept of sub-flow classifier for H.323 packets that can work in conjunction with RLP retransmissions to improve the call set-up time.

\section{REFERENCES}

[1] M. Handley, H. Schulzrinne, E. Schooler, and J. Rosenberg, "SIP: session initiation protocol," Request for Comments (Proposed Standard) 2543, Internet Engineering Task Force, Mar. 1999.

[2] International Telecommunication Union, "Packet based multimedia communications systems," Recommendation H.323, Telecommunication Standardization Sector of ITU, Geneva, Switzerland, Feb. 1998.

[3] H. Schulzrinne, S. Casner, R. Frederick, and V. Jacobson, "RTP: A transport protocol for real-time applications," Request for Comments (Proposed Standard) 1889, Internet Engineering Task Force, Jan. 1996.

[4] International Telecommunication Union, "Media stream packetization and synchronization on non-guaranteed quality of service LANs," Recommendation H.225.0, Telecommunication Standardization Sector of ITU, Geneva, Switzerland, Nov. 1996.

[5] International Telecommunication Union, "Control protocol for multimedia communication," Recommendation H.245, Telecommunication Standardization Sector of ITU, Geneva, Switzerland, Feb. 1998.

[6] G. Bao, "Performance evaluation of TCP/RLP protocol stack over CDMA wireless link," Wireless Networks, No.3, 2 (1996) 229-237.

[7] S. K. Sen, J. Jawanda, K. Basu, N. K. Kakani, S. K. Das, "A Call Admission Control Scheme for TCP/IP based CDMA Voice/Data Network," ACM/IEEE International Conference on Mobile Computing and Networking, Oct 1998, Dallas, pp. 276-283.

[8] J. Postel, "Transmission Control Protocol," Request for Comments 793 , Internet Engineering Task Force, Sept. 1981.

[9] L. Westberg and M. Lindqvist, "Realtime Traffic over Cellular Access Networks," IETF draft-westberg-realtime-cellular-01.txt, Oct. 1999.

[10] S. A. Thomas, IPng and the TCP/IP Protocol, New York: Wiley Computer Publishing, 1996.

11] D. Minoli and E. Minoli, Delivering Voice over IP Networks, New York: Wiley Computer Publishing, 1998.

12] K. Singh and H. Schulzrinne, "Interworking Between SIP/SDP and H.323," IETF draft-singh-sip-h323-00.txt, Jan. 2000. 
APPENDIX: Fast Connect Procedure

Initiation of Fast Connect procedure: The calling endpoint initiates the Fast Connect procedure by sending a H.225 Setup message containing the bffastStart element to the called endpoint. The fastStart element consists of a sequence of H.245 OpenLogicalChannel structures (in order of preference), each describing a media channel which the calling endpoint proposes to send and receive, including all the parameters necessary to immediately open and begin transferring media on the channels.

Refusal of Fast Connect procedure: The called endpoint may refuse to use the Fast Connect procedure, either because it does not implement it or because it intends to invoke features that require use of the procedures defined in Recommendation H.245.

Acceptance of Fast Connect Procedure: When the called endpoint desires to proceed with the Fast Connect procedure, it sends a H.225 message (Call Proceeding, Alerting, Progress or Connect) containing a fastStart element selecting from amongst the openLogicalChannel proposals offered by the calling endpoint. Channels accepted are considered opened as the usual H.245 openLogicalChannel and openLogicalChannelAck procedure has been followed.

Media transmission: The called endpoint may begin transmitting media (according to the channels opened) immediately after sending an H.225 message containing the fastStart element. The calling endpoint must therefore be prepared to receive media on ANY of the receive channels it proposed in the H.225 Setup message, since it is possible for the media to be received prior to the H.225 message indicating precisely which channels to be used. Once an H.225 message containing the fastStart element is received by the calling endpoint, it may discontinue attempting to receive media on the channels for which proposals were not accepted by the called endpoint.

The calling endpoint may begin transmitting media (according to the channels opened) immediately upon receiving an H.225 message containing the fastStart element. Therefore, the called endpoint must be prepared to receive media on the channels it accepted in the H.225 message containing the fastStart element.

Switching to $\mathbf{H . 2 4 5}$ procedures: After establishing a call using Fast Connect procedure, either endpoint may initiate the use of H.245 procedures at any time during the call, using tunneling or a separate H.245 connection.

Terminating a call: If a call connect using the Fast Connect procedure continues to completion without initiation of H.245 procedure, the call may be terminated by either endpoint sending an H.225 Release Complete message.

We assume the simple direct call set-up message flows as described in Figure 8. The TCP level interactions for exchanging the H.225 messages is shown in Figure 9. The associated message sizes are given in Table 1. The total call set-up delay will be the cumulative delay for (1) set-up time for the TCP session (exchange of SYN, SYN-ACK, ACK messages); (2) successfully transmitting all the H.225 messages shown in Figure 3; (3) successfully receiving an RTCP CNAME message.

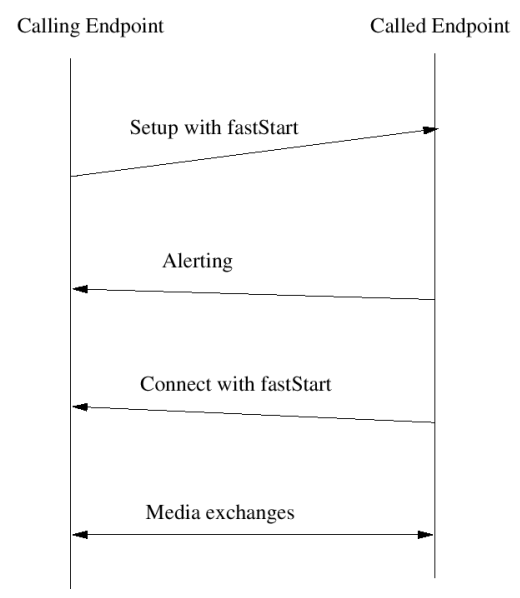

Fig. 8. H.225 Fast Connect Call Signaling

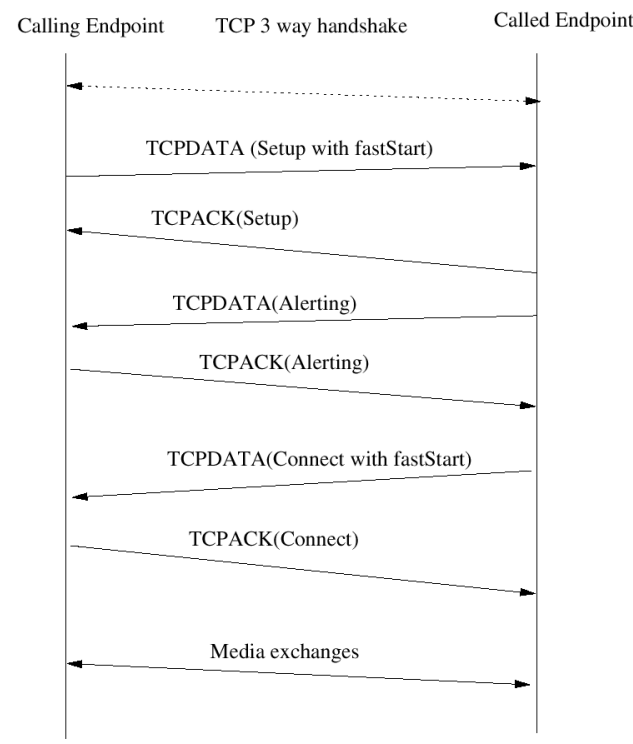

Fig. 9. Fast Connect Call Setup and TCP interactions

Assume that in this Fast Connect procedure the calling endpoint advertises three different media channels and one codec in each openLogicalChannel structure in the fastStart elemen$\mathrm{t}$ of the Setup message. Furthermore, the corresponding response to fastStart is done in Connect message without loss of generality. 